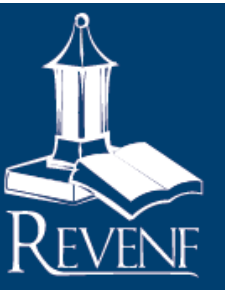

\title{
Programa educativo para la prevención y el manejo de la violencia escolar. ${ }^{1}$
}

\author{
Institución: Universidad de Costa Rica
}

\author{
Viriam Leiva Diaz ${ }^{2}$ \\ Sharon Quiel Castro ${ }^{3}$ \\ Francini Zúñiga Arguedas 4
}

\section{COMO CITAR}

Leiva, V., Quiel, S. y Zuñiga, F. (abril, 2013). Programa educativo para la prevención y el manejo de la violencia escolar. Rev. Actual en Costa

Rica, 23, 1-18. Recuperado de: <http://www.revenf.ucr.ac.cr/violencia.pdf>ISSN 1409-4568

\section{RESUMEN}

Este artículo presenta los principales resultados de la implementación de un programa educativo para la prevención y manejo de la violencia en las escuelas públicas por parte de los maestros de primero y segundo ciclo, el programa fue impartido por la Escuela de Enfermería de la Universidad de Costa Rica, con un total de 40 horas de enero a febrero del 2011. Se utilizó diversas estrategias de enseñanza basadas en las necesidades educativas de este grupo de profesores, las cuales se mostraron en un estudio previo y la aplicación de una evaluación de necesidades. Con la asistencia de 33 profesores, 32 mujeres y un hombre. Del total de participantes, 30 completaron el programa. Los principales resultados son los siguientes: los participantes lograron adquirir, construir o mejorar sus conocimientos sobre la prevención y tratamiento de la violencia escolar, y también aprendieron diversas técnicas o estrategias para la prevención y control de la violencia en las escuelas. Se concluye que el éxito en la consecución de los objetivos planteados para cada una de las sesiones se relaciona directamente con el hecho de que todo el programa educativo se apegó a las necesidades educativas expuestas por la población participante y a sus características como docentes, haciendo uso de los principios de andragogía, que permitieron entender el aprendizaje como un intercambio de conocimientos entre las partes involucradas.

Palabras claves: violencia escolar, prevención y manejo, programa educativo, educación en salud, Enfermería.

Fecha de recepción: 15 noviembre 2012

Fecha de aceptación: 15 febrero 2013

Profesora catedrática de la Escuela de Enfermería de la Universidad de Costa Rica. Enfermera, psicóloga, Magister en Psicopedagogía, investigadora del Programa de Investigación en enfermería y del Instituto de Investigaciones Filosóficas. Correo electrónico: viriaml@gmail.com / viriam.leiva@ucr.ac.cr

Licenciada en Enfermería de la Universidad de Costa Rica. Correo electrónico: quielcs@gmail.com Licenciada en Enfermería de la Universidad de Costa Rica. Correo electrónico: gabita0987@hotmail.com 


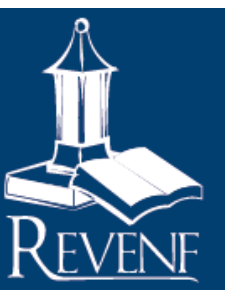

\title{
Educational program for the prevention and management of school violence ${ }^{1}$
}

\author{
Institution: University of Costa Rica
}

\author{
Viriam Leiva Diaz ${ }^{2}$ \\ Sharon Quiel Castro ${ }^{3}$ \\ Francini Zúñiga Arguedas ${ }^{4}$
}

\section{CITED}

Leiva, V., Quiel, S. y Zuñiga, F. (april, 2013). Educational program for the prevention and management of school violence. Rev. Actual en Costa Rica, 23, 1-18 Recuperado de: <http://www.revenf.ucr.ac.cr/violencia.pdf> ISSN 1409-4568

\section{ABSTRACT}

This article presents the main results of the implementation of an educational program for the prevention and management of violence in public schools by teachers of first and second cycle, the program was taught by the School of Nursing at the University of Costa Rica, with a total of 40 hours from January to February 2011. We used various teaching strategies based on the educational needs of this group of teachers, which were shown in a previous study and application of a needs assessment. Attended by 33 teachers, 32 women and one man. Of the participants, 30 completed the program. The main results are as follows: participants were able to acquire, build or improve their knowledge about the prevention and treatment of school violence, and also learned various techniques and strategies for prevention and control of violence in schools. It is concluded that success in achieving the goals set for each of the sessions is directly related to the fact that the entire educational program stuck to the educational needs expressed by the participating population and its characteristics as teachers, using principles of andragogy, which allowed understanding learning as a knowledge sharing among stakeholders.

Key words: education-program, health-education, nursing, prevention-and-management, school violence.

\footnotetext{
${ }^{1}$ Date of receipt: November 20, 2012

Date of acceptance: January 15, 2013

${ }^{2}$ Professor School of Nursing at the University of Costa Rica. Nurse, psychologist, Master in Psychology, researcher Nursing Research Program and the Institute for Philosophical Research. email: viriaml@gmail.com / viriam.leiva@ucr.ac.cr

${ }^{3}$ Graduate in Nursing at the University of Costa Rica. email: quielcs@gmail.com

${ }^{4}$ Graduate in Nursing at the University of Costa Rica. email: gabita0987@hotmail.com
} 


\section{INTRODUCCIÓN}

El fenómeno de la violencia es un problema de antaño que continua afectando a la sociedad tanto en el ámbito internacional como nacional (Blanco, 2007).

De acuerdo con un informe realizado por el Ministerio de Salud de Costa Rica, la Organización Panamericana de la Salud (OPS) y la Organización Mundial de la Salud (OMS) (2003), la violencia debe tratarse como un problema de salud pública, ya que produce lesiones y muertes prematuras, además de fomentar el deterioro de las relaciones sociales en diversas direcciones.

En efecto, la violencia es un fenómeno social que daña a las personas, tanto en el ámbito individual como colectivo y el cual, lamentablemente, se presenta día tras día. Basta con oír o ver los noticieros nacionales e internacionales para percatarse de esta realidad.

Por lo tanto, no es de extrañar que en el decimosexto Informe del Estado de la Nación (Programa Estado de la Nación, 2010) se mencione el hecho de que Costa Rica ha dejado de ser un país con bajos índices de violencia; por el contrario, estos aumentan año tras año. Además, de acuerdo con el Ministerio de Salud, la OPS y el OMS (2004), desde el año 2004 la violencia ya se encontraba entre las principales causas de muerte en Costa Rica.

Sumado a lo anterior, en el Plan Nacional para la Prevención de la Violencia y Promoción de la Paz Social, del Ministerio de Justicia (2010), se presenta, entre otros datos, que el $78 \%$ de la población costarricense consideró al país como inseguro; del año 1990 al 2006, la agresión incrementó en un 100\%; la violación, en un $70 \%$ y el homicidio doloso, en un 50\%. También, se menciona cómo los problemas familiares son características predominantes de los jóvenes involucrados activamente en pandillas. De estos, más del 50\% señala la existencia de situaciones de violencia, agresión y castigos entre los miembros del grupo familiar.

Por tanto, cualquier esfuerzo que se realice de forma planificada y coherente con la realidad, con el fin de combatir este flagelo social en alguna de sus manifestaciones, será de gran valor para evitar o disminuir el daño que provoca la violencia en la sociedad. (Blanco, 2007)

De acuerdo con los autores Yurbero (1996), Asociación pro derechos humanos (1999), Sánchez y Valencia (2007) y Armas (2007), entre otros, un gran porcentaje de los factores causantes de la violencia en la sociedad tiene sus orígenes, principalmente, en las vivencias o en la formación que reciben las personas durante su niñez, ya sea en el ámbito familiar, comunal o escolar.

Relacionado con lo anterior, y a raíz de los resultados obtenidos en una investigación realizada por Alfaro, Kenton y Leiva (2010) acerca de los conocimientos y percepciones sobre la violencia escolar que poseía un grupo de docentes de escuelas públicas de la Dirección Regional de San José de Costa Rica, se gestó el presente estudio como una forma de dar respuesta a las necesidades encontradas. A la vez, pretende aportar conocimientos teóricoprácticos, ideas y esfuerzos para luchar en contra de una de las manifestaciones de este problema social: la violencia escolar. 
Dentro de las insuficiencias encontradas en la población estudiada en la investigación de Alfaro et al. (2010) es posible mencionar la necesidad de recibir una capacitación acerca del tema de prevención y manejo de la violencia escolar. Esto último fue expresado por un $97 \%$ de la población estudiada, lo cual, junto a un diagnóstico de necesidades educativas realizadas por las autoras del presente artículo, motivó a la confección y ejecución del programa educativo, con el fin de brindarles información útil y veraz. De igual manera, incentivó la creación de herramientas prácticas que les permitan prevenir y/o manejar las diferentes expresiones de violencia en las escuelas, con especial énfasis en las que ocurren entre la población escolar.

A pesar de la existencia de estrategias para enfrentar la violencia escolar, tales como la ejecución de programas para la prevención y manejo de la violencia escolar dirigido a escolares, docentes y padres de familia, y el fortalecimiento de factores protectores, escasean las investigaciones, en el entorno nacional, en las cuales se ponen en práctica estos tipos de estrategias. (Alfaro y Kenton (2008), Solano (2010) y Cabezas, (2008).

Por lo tanto, el interés por aportar a la lucha contra la violencia, considerada un problema de salud pública según el Ministerio de Salud, OPS y OMS (2004), sumado a los escasos programas o trabajos en materia de prevención y manejo de la violencia escolar en el nivel nacional Alfaro et al.(2010), Solano (2010) y Cabezas, (2010), los resultados de la aplicación del diagnóstico de necesidades, sin olvidar los valiosos aportes realizados por Alfaro et al., propiciaron en las autoras la iniciativa de atender la necesidad urgente y real que evidenciaron los sujetos de estudio de la investigación mencionada.

Este estudio tuvo como objetivo desarrollar un programa educativo para la prevención y el manejo de la violencia escolar, dirigido a las personas docentes de primero y segundo ciclo que laboran en las escuelas públicas del Circuito 03, Dirección Regional de San José, 2011.

\section{MATERIALES Y MÉTODOS}

La confección del diagnóstico del programa educativo se basó en el enfoque cuantitativo descriptivo, el cual, de acuerdo con Barrantes (2003), “... se fundamenta en los aspectos observables y susceptibles de cuantificar" (Barrantes, 2003, p.64). Esto permitió comparar las bases bibliográficas encontradas con el estudio de la población docente encuestada sin interferir en el acontecimiento dado que correspondió al diagnóstico de necesidades.

Además, para lograr la consecución del objetivo propuesto del proceso educativo, las investigadoras se fundamentaron en los objetivos planteados. Estos permitieron establecer cinco fases descritas a continuación:

Fase de Coordinación: Inició con la respectiva revisión bibliográfica y delimitación del tema. Incluyó las gestiones realizadas para la aprobación del proyecto, por parte del las autoridades del Circuito 03 y de la Comisión de Trabajos finales de Graduación, además de la inscripción del trabajo al Programa de Extensión Docente de la Universidad de Costa Rica.

Fase Diagnóstica: Durante esta etapa se identificaron las necesidades educativas de la población meta mediante un instrumento de recolección de información. 
Fase de Diseño del Programa Educativo: Con base en la información recolectada y el análisis de esta, se elaboró el programa educativo.

Fase de ejecución del Programa Educativo: Luego de elaborar dicho programa, se llevó a cabo cada una de las sesiones planteadas, en el edificio de la Escuela de Enfermería de la Universidad de Costa Rica, con la participación de 33 docentes matriculados. Dichas sesiones se basaron en los principios andragógicos y constructivistas, los cuales permitieron desarrollar el programa educativo de acuerdo con as características del proceso de aprendizaje de las personas adultas (Requejo, 2003).

Fase de evaluación del proceso: Finalmente, se realizó un proceso de evaluación, en donde se valoró qué tan eficiente resultó el desarrollo del programa educativo; esto desde la perspectiva de las personas participantes. Se utilizaron dos instrumentos diagnósticos, uno elaborado por las facilitadoras y otro confeccionado por la unidad de Extensión docente de la Universidad de Costa Rica.

Respecto a la población que participó en la fase diagnóstica, esta estaba conformada por los 189 docentes que laboraban en las escuelas públicas del circuito 03. El mayor porcentaje de las y los participantes se encontraba en el grupo de los 31 a los 56 años de edad y, en su mayoría, fueron mujeres; solo 6 de los participantes eran hombres. Sin embargo, únicamente 33 docentes participaron en el programa educativo, ya que se estableció un cupo limitado; esto con el fin de facilitar y permitir un desarrollo óptimo de las sesiones establecidas para el programa educativo.

\section{Consideraciones éticas}

Cuando se trabaja con personas, es necesario apegarse a los principios éticos para así garantizar la conservación de sus derechos e integridad (Serrano, 2003). Con base en lo anterior, para el estudio se tomó en consideración principios bioéticos planteados por Blázquez (2004), entre los que se pueden citar los siguientes:

Autonomía: se respetó a las personas como individuos libres y se tuvo en cuenta, en todo momento, sus decisiones.

Veracidad: las personas que participaron fueron informadas verazmente sobre todo lo referente a su participación en el Programa educativo para la prevención y manejo de la violencia escolar.

Privacidad: Se mantuvo el anonimato de los participantes con el fin de velar por el cumplimiento de este principio.

Beneficencia y no maleficencia: El presente trabajo se desarrolló mediante el desarrollo de actividades que no fueran en contra de principios, sentimientos, creencias o integridad de cada participante. Además, además se buscó su beneficio por medio de la construcción de conocimientos respecto al tema de interés de las personas participantes.

\section{RESULTADOS}

Durante la etapa diagnóstica, se buscó conocer las necesidades educativas que poseía la población docente. Estas sirvieron de base para lo que, posteriormente, sería el planteamiento y desarrollo del programa educativo. 
Al desconocer quiénes se inscribirían en el programa educativo, se decidió establecer un diagnóstico y abordar la totalidad de docentes de las escuelas públicas del circuito 03 de la Dirección Regional de San José. Es decir, se consideró a los 189 docentes que laboraban para dichas escuelas; de estos, 121 contestaron a cabalidad el cuestionario autoadministrado del cual se obtuvo los resultados explicados a continuación.

Es importante mencionar en el presente estudio que, en la fase diagnóstica, se encuestó -según la información suministrada por los centros educativos- a la misma población que fue investigada por Alfaro et al. (2010), lo que permite hacer comparaciones y validar los resultados obtenidos en dicho trabajo.

En cuanto a los aspectos sociodemográficos, el $94 \%$ de la población correspondió al sexo femenino y casi el $75 \%$ de la población se encontró en el grupo de edades comprendidas entre los 31 años a los 56 años de edad. Sumado a lo anterior, el $58 \%$ indicó encontrarse casada o casado, y un $66 \%$ dijo poseer un grado académico de licenciatura o maestría.

Entrando en el tema de necesidades educativas, se determinó que los docentes consideraron necesario abordar, en el programa educativo, los siguientes tópicos: Prevención de la violencia escolar- según el 98\% de la población- , el manejo de la violencia escolar -de acuerdo al 87\%-. Un $82 \%$ señaló el tema de tipos de enseñanza violenta al igual el de factores protectores en contra de la violencia escolar; seguidos por la resolución alternativa de conflictos, lo que dice el Reglamento del Ministerio de Educación Pública (Ministerio de Educación Pública, 2009) respecto al manejo de la violencia y las características de las personas victimarias, según el $81 \%$ de la población encuestada. Por último, dentro de los 10 temas considerados como necesidades educativas, por la población docente, se encontró: tipos de crianza violenta y caracterización de las personas víctimas de violencia escolar, ambos con un $80 \%$ de aprobación por parte de los docentes.

Por último, respecto a la metodología de preferencia para recibir el programa educativo, más de la mitad de los participantes escogió la modalidad de talleres y charlas, y casi un $70 \%$ indicó que el programa debía tener una duración de entre 1 y 2 horas por sesión.

Los hallazgos anteriores (de la etapa diagnóstica) fungieron como base para la elaboración del programa educativo para la prevención y manejo de la violencia escolar, cuya ejecución arrojó los siguientes resultados.

En total, se realizaron 10 sesiones educativas basadas en las necesidades expresadas por la población docente -tanto en la investigación previa de Alfaro et al. (2010)como en la etapa diagnóstica-. Se contó con la participación de 33 docentes, 32 mujeres y un hombre; 30 de ellos terminaron el programa. A continuación se resume, en términos generales, los principales resultados obtenidos durante la ejecución de las sesiones:

En lo que se respecta a la temática de la construcción social de la violencia en Costa Rica, los participantes construyeron una línea del tiempo que explicó la construcción social de la violencia en Costa Rica; así como una síntesis de las diferentes dimensiones involucradas en el fenómeno de la violencia. Dentro de estas últimas, mencionaron la social, la histórica y la económica de acuerdo con sus conocimientos previos. 
Además, coincidieron en la discusión realizada en señalar la existencia de determinados aspectos como generadores de más violencia en Costa Rica. Entre ellos se mencionaron la desigualdad de género en relación con los estereotipos machistas, la discriminación, la adopción de conductas negativas y las desigualdades económicas.

Se discutió sobre las relaciones de poder que intervienen en la violencia, con experiencias o conocimientos previos, a través del uso de la técnica de la charada (De Natele, 2003). Esta última permitió mostrar diferentes situaciones de violencia y los escenarios donde se desarrollan.

Otro aspecto analizado fue el de los comportamientos de los docentes que pueden ser violentos o generar violencia. Así, los participantes identificaron algunas actitudes o conductas (efectuados por las maestras o maestros) que pueden ser violentas o generar violencia hacia sus alumnos o alumnas. Lo anterior se dio mediante comentarios u opiniones, como las siguientes "En el caso que nos tocó la violencia se dio porque la maestra ignoró la necesidad que tenía la alumna de ir al baño, lo cual provocó que se orinara y fuera víctima de las burlas de sus compañeros", "en el caso de nosotras la violencia se dio porque el maestro golpeó el pupitre y alzó la voz para llamar la atención o mantener la disciplina", "en el de nosotros la violencia se dio porque la maestra agarró al niño del brazo y lo sentó a la fuerza en el pupitre, porque este no le hacía caso". (Participantes, 2011); las y el participante expresaron como en las aulas todavía existen situaciones de violencia por parte del personal docente contra sus discentes.

Cabe destacar una expresión de un docente quien, durante la actividad, dijo lo siguiente: Para mí no toda la violencia es mala, porque antes a uno le jalaban las orejas, le pegaban al pupitre con el borrador y uno hacía caso y lo tenían controlado, en cambio ahora todo es muy permisivo porque no se pueden ni volver a ver los alumnos porque lo acusan de violencia a uno. (Participante, 2011)

Se evidenció que las personas participantes fueron víctimas de violencia por parte de sus docentes en la escuela y cómo aún existe este tipo de actos. Esto resultó evidente en intervenciones como la siguiente: Yo tengo a mi hija en primer grado de escuela, ella es muy activa y su maestra no le tiene paciencia y en muchas ocasiones la ha agredido verbalmente por esto; y cuando yo fui a hablar con ella, también me agredió a mí al decirme que en "casa de herrero cuchillo de palo". Dando a entender que yo no sabía educar a mi hija, siendo yo docente. (Participante, 2011)

Asociado a lo mencionado, se trabajó, por medio de sociodramas, acerca de las características de las personas victimarias y víctimas de violencia escolar. De esta forma, se abrió un espacio de discusión grupal que permitió a las personas docentes participantes expresar en sus comentarios sobre la importancia de conocer sobre el tema. Entre lo comentado se encuentra: Me parece interesante conocer el perfil de los niños víctima y victimarios, ya que uno puede tomar medidas para fortalecer la autoestima y otras cualidades, para disminuir la posibilidad de que surja violencia entre los alumnos. (Participante, 2011)

Uno a veces ve alumnos muy tímidos y que no les gusta jugar con los otros compañeritos, sin percatarse que puede ser víctima de violencia. (Participante, 2011)

Como se nota mediante estas opiniones, las personas participantes indicaron la importancia de identificar estas características para prevenir la violencia. 
Sin embargo, no solo es fundamental conocer sobre las características de las personas inmersas en situaciones de violencia, sino también también capacitarse en el reconocimiento de los tipos de violencia que existen y acerca de lo estipulado en el Reglamento del Ministerio de Educación Pública de Costa Rica para el manejo de esta.

El grupo de participantes emitió opiniones y formuló preguntas o aclaraciones acerca de las definiciones presentadas sobre los diferentes tipos de violencia desarrollados en el ámbito escolar. Además, analizaron, criticaron y propusieron mejoras al Reglamento de Educación Pública, respecto a la forma en que deben actuar los docentes ante situaciones de violencia.

En lo que respecta a los tipos de violencia, es importante destacar lo mencionado por los participantes acerca de los tipos de violencia presentados y discutidos en el programa. Dentro de los comentarios más relevantes, se encuentran los siguientes:

Esta definición que presentan ustedes de violencia física llama la atención al mencionar que es de forma no accidental, porque muchos de los agresores suelen justificarse diciendo que fue sin culpa. (Participante, 2011)

También en la parte de violencia física que dice que puede o no causar lesiones externas, porque uno piensa que solo cuando ve aquel morete es que la persona está siendo víctima de violencia física, pero en esta definición va el jalar el pelo y cosas así que no dejan secuelas visibles y que uno no contemplaba. (Participante, 2011)

El incluir en la violencia psicológica cosas como el no reconocer aciertos de los niños y niñas, la humillación pública o el ridiculizar es importante, ya que muchas veces en las aulas solo vemos las cosas malas que hacen los niños y nunca les alentamos cuando hacen cosas buenas o siempre le estamos diciendo a Fulanito: es que a usted sí le cuesta entender, delante de los demás compañeritos y eso crea en ellos un sentimiento de vergüenza y tal vez uno no lo veía como violencia o hasta lo hace inconscientemente. (Participante, 2011)

Llama especialmente la atención que uno tiene pensado que la violencia escolar es la que se da entre los estudiantes, y en esta definición se menciona que incluye a todo el sistema educativo y ese sistema lo conformamos todos, no solo los alumnos sino también los docentes, los administrativos y hasta los conserjes. (Participante, 2011)

El segundo aspecto analizado fue el Reglamento del Ministerio de Educación Pública de Costa Rica como una herramienta para el manejo de la violencia escolar. De esta profundización, se encontró que los docentes tienen críticas constructivas hacia este; esto es evidente en el siguiente comentario: Si es cierto, muchos docentes preferimos enviar boletas muy leves o leves ante faltas graves, muy graves o gravísimas. Por el proceso engorroso de las faltas graves, muy graves o gravísimas. Imagínese que en muchos casos uno sale hasta demandada porque a los estudiantes se les asignan abogados y uno tiene que defenderse sin ayuda de nadie. (Participante, 2011)

Ante esto, se trabajó sobre alternativas o recomendaciones que darían los y las participantes para mejorar el reglamento. Dentro de estas, las siguientes opiniones engloban las ideas más importantes: 
Solicitar al MEP, que en el reglamento de evaluaciones se establezca claramente el tipo de acciones correctivas según la falta cometida, para evitar reclamos o apelaciones por parte de los estudiantes o padres de familia. (Participante, 2011)

Sugerir a los directores o directoras que el reglamento interno de cada institución se entregue a los encargados de los estudiantes, para que lo lean, lo conozcan y lo firmen aceptándolo. (Participante, 2011)

Dentro de la violencia escolar, destaca un fenómeno único en dicho ambiente: el "Bullying". Es de suma importancia que los docentes conceptualicen e identifiquen claramente sus características para poder intervenir de forma pronta y oportuna. Cabe destacar que las personas participantes desconocían acerca del término o estaban confusos sobre su definición. Incluso, una docente expresó "No me queda claro por qué el 'bullying' es algo exclusivo de los escolares" (Participantes, 2011). Lo anterior guió la discusión y las estrategias educativas para que los y las docentes construyeran un concepto propio que englobara el fenómeno del "Bullying". Un comentario que muestra dicha construcción fue la manifestada por dos de las participantes: Ya me quedó claro que no es lo mismo la violencia entre escolares que el bullying, porque la violencia entre escolares puede ser cualquier evento aislado o repentino, mientras que el bullying es un coso repetitivo, planeado y que se da a lo largo del tiempo. (Participante, 2011)

Bueno, el fenómeno del bullying trae graves consecuencias tanto para las víctimas como victimarios, porque les afecta el autoestima y su capacidad de relacionarse o desarrollarse en la sociedad. A futuro este tipo de personas pueden caer en depresión o llegar a actos delictivos por su incapacidad de relación social. (Participante, 2011)

El reconocimiento de los tipos de violencia, así como el fenómeno "bullying" para una detección precoz, es fundamental, pero lo es también el aprender estrategias de intervención; de lo contrario, no excedería la mera teoría, lo cual imposiblilitaría la respuesta por parte de los y las docentes. Por esta razón, dentro de la capacitación se realizó una sesión que pudiese contestar a la pregunta: ¿Qué hacer ante una situación de violencia escolar? Para esto, se hizo uso de los pasos de intervención que proponen Varela, Tijmes y Sprague (2009). La forma en que fueron asimilados por los docentes queda explicitada en la siguiente tabla resumen. 


\section{Revista Electrónica Enfermería Actual en costa Rica}

Tabla 1: Resumen de la construcción práctica de la intervención en situaciones de violencia escolar por parte de las personas participantes, según los pasos planteados por Varela, Tijmes y Sprague (2009)

\begin{tabular}{|c|c|}
\hline Pasos & $\begin{array}{l}\text { Conceptualización de las personas } \\
\text { participantes }\end{array}$ \\
\hline $\begin{array}{l}\text { "Actuar calmadamente, no de forma } \\
\text { amenazante" y ayude a su estudiante a } \\
\text { calmarse". }\end{array}$ & $\begin{array}{l}\text { "Nos parece importante estos puntos, } \\
\text { porque por lo general los maestros y } \\
\text { maestras actuan histéricamente ante } \\
\text { manifestaciones de violencia entre los } \\
\text { escolares, porque a uno le da enojo. Pero al } \\
\text { tratar de calmarse ante estas situaciones le } \\
\text { permite a uno pensar mejor las cosas para } \\
\text { actuar de la mejor forma posible". "La } \\
\text { segunda frase es parecida, si uno logra } \\
\text { tranquilizar al estudiante, será más fácil } \\
\text { intervenir o dialogar con él". (Participantes, } \\
\text { 2011) }\end{array}$ \\
\hline $\begin{array}{l}\text { "Recordar el comportamiento que se espera } \\
\text { del alumno o alumna" y conocer el motivo: } \\
\text { Preguntarle por qué realizó el acto de } \\
\text { violencia". }\end{array}$ & $\begin{array}{l}\text { "La primera estrategia, permite que el } \\
\text { alumno o alumna caiga en razón de que lo } \\
\text { que hizo estuvo mal y no es lo que se } \\
\text { esperaba de él o ella, es como para activar su } \\
\text { consciencia, pensamos nosotros. Y la } \\
\text { segunda estrategia es importante para no } \\
\text { juzgar sin conocer primero lo que sucedió y } \\
\text { de esta forma evitar malos entendidos". } \\
\text { (Participantes, 2011) }\end{array}$ \\
\hline $\begin{array}{l}\text { "Fomentar la empatía, buscar una solución } \\
\text { que satisfaga a todos y reflexionar acerca de } \\
\text { lo ocurrido". }\end{array}$ & $\begin{array}{l}\text { "Es importante que nosotros nos pongamos } \\
\text { en los zapatos de los niños para lograr } \\
\text { comprender su comportamiento", "Aunque } \\
\text { sea dificil, lo mejor es buscar una solución } \\
\text { neutral para evitar resentimientos o } \\
\text { rivalidades, y como es obvio, siempre es } \\
\text { importante reflexionar o meditar acerca de } \\
\text { nuestros comportamientos, y poner a } \\
\text { reflexionar a los niños o niñas que realizan } \\
\text { hechos violentos acerca de las consecuencias } \\
\text { de sus actos, para que no vuelvan a } \\
\text { cometerlos, o evitar las situaciones que los } \\
\text { detonan". (Participantes, 2011) }\end{array}$ \\
\hline
\end{tabular}


Como complemento de lo expuesto en la tabla resumen, se analizó, con los y las participantes, la estrategia denominada Resolución alternativa del conflicto. Los elementos de esta son: la negociación, la mediación, la conciliación y el arbitraje. Para cumplir con lo anterior, se hizo uso del análisis de casos; un ejemplo de la forma analizada por las personas participantes fue el siguiente:

En este caso le explicaríamos a los dos niños los pasos para solucionar alternativamente el conflicto y que en primer lugar deben conversar a ver si pueden llegar a una solución por ellos mismos, si no lo logran les diríamos que busquen alguna persona de confianza que les ayude a entender el punto de vista de cada uno a ver sí de esta forma logran llegar a un acuerdo, sino es así les diríamos que pidan consejos o soluciones a alguna persona de confianza para los dos a ver si logran solucionar el problema. Y si no lo logran les diríamos que en última instancia nosotros los docentes tomaríamos la decisión de quien se queda en el lugar que los dos querían estar, que yo pienso que lo más salomónico sería que ninguno de los dos se quedara en ese lugar, y más bien sentar a otro niño ahí. (Participante, 2011)

Por último, a raíz de la aplicación de los instrumentos diagnósticos en la sesión número diez, se obtuvo como principales resultados que la población participante calificó el programa, en general, como "muy bueno". Esto incluyó los materiales y métodos empleados y el desenvolvimiento de las facilitadoras del programa educativo.

\section{DISCUSIÓN}

La etapa diagnóstica arrojó datos importantes para la construcción del programa educativo, ya que se tomaron en cuenta para su confección aspectos demográficos tales como el sexo que, de acuerdo con Salas (2008), es relevante pues el proceso de aprendizaje de las mujeres (sexo predominante de la etapa diagnóstica) y la edad de las personas docentes, pudieron dirigir el programa hacia los principios o métodos andragógicos que se encuentran enfocados en "ayudar a los adultos a aprender" (Requejo, 2003). También fue importante el grado académico de las personas docentes, el cual permitió prever, desde el punto de vista andragógico (Carreras, 2003), que quienes participarían del programa educativo lo harían con el fin de enriquecer su cultura y actualizar o acrecentar los conocimientos que ya poseen. También, participarían con el fin de dinamizar su experiencia, mejorar profesionalmente o simplemente para satisfacer necesidades o intereses de su vida diaria (López y Sarrate, 2002).

La comprensión y el análisis de las características de los modelos de crianza: el democrático, el permisivo, el autoritario y el negligente, mencionados por Estalayo, Rodríguez y Romero (2009), son fundamentales en cualquier proceso de capacitación de personas responsables del cuidado y educación de niños y niñas. Esto último porque pone a disposición información útil para brindar consejos o recomendaciones a los padres y las madres acerca de los métodos de crianza más beneficiosos para sus hijos e hijas, formas de evitar conductas violentas o el peligro de que se conviertan en víctimas de agresión (Serrano e Iborro, 2005). De esta forma, también se podrán convertir enseres promotores de la educación en salud atacando la violencia que puede darse en los hogares y por ende las consecuencias negativas en la salud física y mental de las personas involucradas (Blanco y Maya, 2006). 
Analizar las estructuras de poder es relevante, ya que permite comprender su dinámica. Destaca que, en toda trato humano, se establecen relaciones de poder que no son malas per se, sino por la manera en la que se manifiestan. Tal y como lo apuntan Gaxiola y Frías $(2008$, p.), una relación de poder, si no se sabe manejar adecuadamente, puede llegar a convertirse en una situación de violencia en diferentes ámbitos (familiar, comunal, escolar, laboral, otros). Esta comprensión es fundamental para el personal docente, pues le permite identificar las diferentes relaciones de poder existentes en situaciones de violencia y los escenarios donde estas se desarrollan.

La identificación de actos de violencia no siempre es clara y, en muchos casos, sucede lo manifestado por Castells, (2007), Blanco (2007) y Jiménez (2007), quienes indican cómo en muchas ocasiones existe una "normalización" y aprendizaje de la violencia, es decir, la violencia es aprendida socialmente y de igual forma enseñada como algo normal o necesario para alcanzar un fin. Sin embargo, la violencia siempre genera más violencia (Alda y Beliz, 2007); por consiguiente, más problemas de índole social se ven reflejados en daños físicos, psicológicos, e inclusive muertes, en el peor de los casos (Jiménez, 2007). Por esto, se debe luchar para evitar naturalizar la violencia.

Unido a lo anterior, las consecuencias negativas de cualquier acto de violencia sobre la vida de una persona y el hecho de que cause temor y sumisión en los escolares no implica que sea la forma adecuada para mantener el orden, ya que existen métodos pedagógicos útiles y en especial seguros para este fin (Marí, 2006). Es importante recordar, en este punto, que de acuerdo con los resultados de Alfaro y Kenton (2008) se evidenció cómo algunos y algunas docentes incurrían a actos violentos contra sus alumnos y alumnas. Arias, Feoli y Fernández (2001) mencionan que algunas de esas actitudes u omisiones pueden ser: "imponer tareas arbitrarias, subestimar o sobreestimar la capacidad de sus alumnos, utilizar el miedo para imponer disciplina, los golpes físicos, gritos, indiferencias, humillaciones, menosprecio, sobreprotección, entre otras" (p.25).

De acuerdo con Defrance (2005), el principal motivo por el cual las personas docentes recurren a la violencia de cualquier índole contra sus estudiantes es no saber cómo instituir el orden y la disciplina. Lo anterior, según el mismo autor, se puede lograr si utilizan sus conocimientos pedagógicos. Por lo tanto, capacitar a las personas docentes posibilita la exploración crítica en la temática y permite proponer herramientas efectivas para el mejoramiento de las actitudes docentes en la enseñanza, de tal forma que esta no llegue a ser violenta contra los discentes.

De igual forma, no se puede intervenir en situaciones que generan violencia si no se reconoce el tipo adecuadamente; en lo que respecta a esto, Sagot (2000) define la violencia física como un acto intencional que puede provocar o no lesiones visibles. Según los participantes, esta última es la más reconocida. Lo anterior no fue tan evidente al definir otros tipos de violencia, tales como la psicológica, la sexual y la escolar. Con excepción de la primera de estas, los insultos, los desprecios, las burlas o las críticas como formas de violencia; de igual modo, se logró, por parte de los participantes, ampliar el concepto de violencia sexual la exposición a material pornográfico o el acoso psicológico sexual (Ramos, 2005), de igual forma la violencia escolar que incluye no solo la relación entre los discentes sino cualquier episodios de hostilidad, agresión, maltrato o discriminación generado en el sistema educativo (Osorio, 2006), que incluye no solo los alumnos sino tanto entre docentes, administrativos y personal misceláneo. 
Dentro de los tipos de violencia, uno en especial debía ser conocido por parte del personal docente para su adecuada intervención: el fenómeno "Bullying" que, de acuerdo con Olweus (1998) y Beane (2006), es una serie de actos de violencia planeados durante un largo periodo de tiempo y que ocurre exclusivamente entre escolares.

Todo este marco conceptual es importante, pero insuficiente para que el personal docente pueda intervenir. Se necesita de herramientas prácticas para su accionar en la cotidianidad; por esto, el primer paso es discutir acerca de la legislación o regulación con la que cuentan los y las docentes. En este caso, se trata del Reglamento de evaluación del MEP (2009), sin embargo, los participantes mostraron poco conocimiento acerca del documento, a la vez que lo calificaron de poco práctico.Esto úlimo resulta preocupante, ya que las sanciones de faltas estipuladas como graves, muy graves o gravísimas son un tipo de medida que, en última instancia, resultan necesarias para controlar o manejar las situaciones de violencia: esto en aquellos casos donde no hubo una respuesta positiva ante otros tipos de intervenciones (Hernández y Sancho, 2004).

La resolución alternativa de conflictos es una opción creativa que puede ser aplicada por los profesionales en Educación. Se trata de un proceso que permite solucionar disputas o diferencias, de una forma pacífica, haciendo uso del diálogo (Vinyamata, 2005) y de los métodos para la prevención y el manejo de la violencia escolar. Estos últimos se logran mediante la elaboración y ejecución, en conjunto, de programas dirigidos a los padres, estudiantes y docentes de los centros escolares (Márquez, 2009).

Varela et al. (2009) indican la importancia de que el docente utilice estrategias específicas para mejorar la resolución de conflictos. Entre estas se menciona el utilizar un tono de voz calmado, al dirigirse al niño o la niña que cometió el acto de violencia, viéndole a los ojos. Además, se señala la conveniencia de controlar el enojo ante la situación, pues esto permitirá manejar adecuadamente el problema sin recurrir a gritos, insultos o alguna forma de agresión.

En relación con lo anterior, otra propuesta es la escucha activa, la cual consiste en prestar máxima atención tanto a lo expresado verbalmente como a las conductas no verbales. Estas últimas aportan información útil sobre los sentimientos, estado de ánimo y preocupaciones de la persona que está hablando (Peplau, 1990).

Como apuntan Varela et al. (2009), esta estrategia pretende que los docentes ayuden al infante a entender cómo sus actos afectan a la persona víctima y cómo se puede haber sentido esta. De esta forma, se fomenta la empatía para propiciar el arrepentimiento de las acciones cometidas.

Los autores antes citados mencionan que la mejor forma de corregir los problemas provocados por violencia es buscar una solución, conjuntamente, de tal forma que se favorezca a todas las partes involucradas. Esto se logra, por ejemplo, haciendo uso de la resolución alternativa de conflictos. Lo anterior coincide con lo expuesto por Bisquerra (2008), quien indica que los factores protectores contra la violencia escolar son: entornos escolares positivos como espacios recreativos de esparcimiento y supervisados, el apoyo grupal o individual como el fomento de valores y habilidades sociales, la aplicación de límites y expectativas claras, el uso constructivo del tiempo, entre otros. Esto último resulta importante, desde el enfoque de educación en salud, ya que las personas participantes adquirieron información útil que les permitirá tomar decisiones para mejorar la condiciones de sus centros educativos o fomentar las habilidades para la vida de sus escolares con el fin de contribuir en controlar o mantener la salud e integridad de las personas (Vargas, 2006) 
El éxito en la consecución de los objetivos planteados para cada una de las sesiones se relaciona directamente con el hecho de que todo el programa educativo se apegó a las necesidades educativas expuestas por la población participante y a sus características como docentes, haciendo uso de los principios de andragogía, que permitieron entender el aprendizaje como un intercambio de conocimientos entre las partes involucradas, con el fin de propiciar un crecimiento personal (Carreras, 2003). Además de seguir un enfoque constructivista que enfoca el proceso de aprendizaje en las personas participantes, y fomentarles una reestructuración interna de los conocimientos previos con los nuevos (Díaz, 2002).

Por último, cabe mencionar que la utilización de la modalidad de taller durante las sesiones facilitó que las personas docentes trabajaran en pequeños grupos para así desarrollar las temáticas planteadas cada día de forma participativa (Fernández, 2008). Además, mediante diversas actividades, se fomentó la unión del grupo, la retroalimentación y el fortalecimiento del conocimiento adquirido.

Todo lo hasta aquí mencionado permite entender la buena calificación que la población participante, durante la fase de evaluación, dio, en términos generales, al desarrollo y coordinación del programa educativo para la prevención y manejo de la violencia escolar.

\section{CONCLUSIONES}

Con base en los resultados obtenidos durante el desarrollo de la investigación, es posible plantear las siguientes conclusiones:

Se evidenció que las personas docentes adquirieron, construyeron o mejoraron su conocimiento en relación con la prevención y el manejo de la violencia escolar, además de las diversas técnicas o estrategias que existen para esto.

La aplicación del instrumento evaluativo, emitido por la Vicerrectoría de Acción Social de la Universidad de Costa Rica, permitió constatar que el programa educativo para la prevención y manejo de la violencia escolar dirigido a docentes cumplió con los objetivos planteados, las expectativas y necesidades educativas de las personas participantes. Además, se empleó la metodología y técnicas apropiadas para exponer las temáticas, esto sin omitir que el espacio físico donde se desarrolló fue el conveniente y el desempeño de las facilitadoras se consideró como óptimo.

La andragogía, el constructivismo y la metodología participativa fueron transcendentales para el desarrollo del curso, pues facilitaron el proceso educativo. En este último, cada participante pudo construir o mejorar sus conocimientos respecto a la violencia escolar y a las herramientas para la prevención y el manejo de esta. 


\section{REFERENCIAS}

Alda, E \& Beliz, G. (2007). Cuál es la Salida: La Agenda Inconclusa de la Seguridad Ciudadana. New York, USA: IDB

Alfaro, I., \& Kenton, K. (2008). Conocimientos y percepciones sobre la violencia en los centros educativos que poseen los y las docentes de primer y segundo ciclos que laboran en las escuelas del Circuito 03, Dirección Regional de San José, de Marzo a octubre de 2008. Tesis para optar por el grado de Licenciatura en Enfermería. Universidad de Costa Rica, San José.

Alfaro M, Ivannia.; Kenton P., Karen.; Leiva D., Viriam.(2010)Conocimientos y percepciones del profesorado sobre violencia en los centros educativos públicos.Enfermería Actual en Costa Rica, volumen(18) Recuperado de World Wide Web: http://www.revenf.ucr.ac.cr/violencia.pdf. ISSN 1409-4568

Asociación Pro Derechos Humanos de España, Colectivo Abierto de Sociología. (1999). La violencia familiar: actitudes y representaciones sociales. Primera Edición. Caracas, Venezuela: Fundamentos.

Arias, M., Feoli, D y Fernández, M. (2001). Interacciones Violentas Educador-Educando en el Aula y en el Ámbito Escolar. (Maestría en Psicopedagogía). Universidad Estatal a Distancia, San José, Costa Rica.

Armas, M. (2007).Prevención e Intervención ante Problemas de Consulta: Estrategias para Centros Educativos y Familias. Primera Edición. Madrid, España: WK Educación.

Barrantes, R. (2003). Investigación: Un Camino al Conocimiento. Un Enfoque Cuantitativo y Cualitativo. Costa Rica: EUNEDBeane, A. (2006). Bullying: aulas libres de acoso. Primera Edición. Barcelona, España: Editorial Grao.

Bisquerra, R. (2008). Educación para la Ciudadanía y Convivencia: El Enfoque de la Educación Emocional. Primera Edición. Madrid, España: WK Educación.

Blanco, A. (2007). Convivir con la violencia: Un análisis desde la psicología y la educación de la violencia en nuestra sociedad. La Mancha, España: Universidad de Castilla.

Blanco, J \& Maya, J. (2006). Salud Pública. Segunda Edición. Bogotá, Colombia: Corporación para Investigaciones Biológicas.

Blázquez, N. (2004). La Bioética y los Hijos del Futuro. Madrid, España: Visión NET

Cabezas, H. (2010). ¿Qué ocurre en el aula Costarricense? Los Niños y las Niñas que Maltratan a sus Compañeros. Actualidades Investigativas en Educación, 10(3), 1-21. Costa Rica.Recuperado de http://revista.inie.ucr.ac.cr/articulos/3-2010/archivos/maltrato.pdf

Cabezas. C. (2008). Violencia Escolar: El acoso del profesor hacia el Alumno. Estados Unidos: Editorial Create Space. 
Carreras, C. (2003). Aprender a formar: educación y procesos formativos. Barcelona, España: Paidós Ibérica, S.A..

Castells, P. (2007).Víctimas y matones: claves para afrontar la violencia en niños y jóvenes. Barcelona, España: CEAC.

De Natale, M. (2003). La Edad Adulta, una Nueva Etapa para Educarse. Madrid, España: Narcea S.A..

Defrance, B. (2005). Disciplina en la Escuela. Quinta Edición. Madrid, España: Ediciones Morata.

Díaz, F. (2002). Didáctica y Currículo: Un Enfoque Constructivista. La Mancha, España: Ediciones de la Universidad de Castilla.

Estalayo, A., Rodríguez, O \& Romero, J. (2009). Estilos de Crianza y Ambientes Familiares en Menores y Jóvenes Violentos. Un Modelo Psicoterapéutico de Apoyo Para la Intervención. Cuadernos de psiquiatría y psicoterapia del niño y del adolescente. 48, 113-129. Recuperado de www.elportaldelaconciliacion.com/.../pr_00138_estilos-crianza-jovenes-violentos.pdf

Fernández, F. (2008). ¿Por qué trabajamos? El Trabajo entre el Estrés y la Felicidad. España: Díaz de Santos S.A

Gaxiola, J \& Frías, M. (2008). Un Modelo Ecológico de Factores Protectores del Abuso Infantil: Un Estudio con Madres Mexicanas. Medio Ambiente y Comportamiento Humano, 9(1y2), 13-31.Recuperado de http://webpages.ull.es/users/mach/PDFS/Vol9_1y2/Vol9_1y2_b.pdf

Hernández, F \& Sancho. (2004). El Clima Escolar en los Centros de Secundaria: Mas Allá de los Tópicos. España: Ministerio de Educación

Jiménez, M. (2007). Subversión de la violencia. Primera Edición. México: Universidad Autónoma de México.

López, E \& Sarrate, M. (2002). La Educación de las Personas Adultas: Reto de Nuestro Tiempo. Madrid, España: Dykinson.

Marí, R, (2006). Diagnostico Pedagógico: Un Modelo para la Intervención en Psicopedagogía. Segunda Edición, Barcelona, España: Ariel S.A.

Márquez, M. (2009). La Prevención de la Violencia Escolar. Innovación y Experiencias Educativas Recuperado de:

http://www.csicsif.es/andalucia/modules/mod_ense/revista/pdf/Numero_17/MARIA\%20DEL\%20ROSARIO_MARQUEZ_2.pd $\underline{f}$

Ministerio de Educación Pública. (2009). Reglamento de Evaluación de los aprendizajes. Decreto Ejecutivo No 35355-MEP. San José, Costa Rica. La Gaceta $\mathrm{N}^{\circ}$ 135. Recuperado de http://www.apse.or.cr/webapse/legdoc/leg03.htm 
Ministerio de Justicia. (2010). Plan nacional para la Prevención de la violencia y la promoción de la paz social. San José, Costa Rica. Recuperado de http://www.ocavi.com/docs files/file 329.pdf

Ministerio de Salud de Costa Rica (MS), Organización Panamericana de la Salud (OPS) \& Organización Mundial de la Salud (OMS). (2004). La violencia social en Costa Rica. Serie Análisis de Situación de Salud; No 9. San José: Costa Rica.Recuperado de http:/www.bvs.sa.cr/php/situacion/violencia.pdf

Olweus, D. (1998). Conductas de Acoso y Amenaza entre Escolares. Primera Edición. Madrid, España: Ediciones Morata.

Organización Panamericana de la Salud., Oficina Sanitaria Panamericana y Oficina Regional de la Organización Mundial de la Salud. (2003). Informe Mundial sobre la Violencia y la Salud. Publicación Científica y Técnica No 588. Washington D C, USA. Recuperado de http://www.mex.opsoms.org/contenido/cd violencia/documentos/informemundial_completo.pdf

Osorio. F. (2006). Violencia en las Escuelas: Un Análisis desde la Subjetividad. Primera Edición. Buenos Aires, Argentina: Ediciones Novedades Educativas.

Peplau, H. (1990). Relaciones Interpersonales en Enfermería: Un Marco Conceptual para la Enfermería Psicodinámica. Barcelona, España: Salvat Editores.

Programa Estado de la Nación. (2010). Décimo Sexto Informe Estado de la Nación en Desarrollo Humano Sostenible. San José, Costa Rica. Recuperado de http://www.estadonacion.or.cr/index.php/bibliotecavirtual/costa-rica/estado-de-la-nacion/sinopsis/informe-xvi

Ramos, M. (2005). Manual sobre Violencia Familiar y Sexual. Perú: Flora Tristán

Requejo, A. (2003). Educación Permanente y Educación de Adultos. Primera Edición. Barcelona, España: Ariel S.A

Sagot, M. (2000). Ruta Crítica de las Mujeres Afectadas por la Violencia Intrafamiliar en América Latina: Estudios de Caso de Diez Países. OPS/OMS, Programa Mujer, Salud y Desarrollo. Pan American Health Org.

Salas, R. (2008). Estilos de Aprendizaje a la Luz de la Neurociencia. Primera Edición. Bogotá, Colombia: Coop, Editorial Magisterio.

Sánchez, M \& Valencia, S. (2007). Lectura Sistémica Sobre Familia y el Patrón de la Violencia. Primera Edición. Bogotá, Colombia: Universidad de Caldas.

Serrano, M. (2003).La educación para la salud del siglo XXI: Comunicación y salud. Segunda Edición. Madrid, España: Díaz de Santos.

Serrano, A \& Iborra, I. (2005). Violencia entre Compañeros en la Escuela. España: Goaprint.

Solano, I. (2010). Programa de Educación para la Paz y Resolución Pacífica de Conflictos en Costa Rica. Costa Rica: Universidad de Granada. 


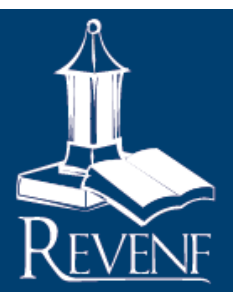

Revista Electrónica Enfermería Actual en costa Rica

Vinyamata, E. (2005). Conflictología: Curso de Resolución de Conflictos. Segunda Edición. Barcelona, España: Ariel S.A.

Varela, J., Tijmes, C \& Sprague, J. (2009). Paz Educa: Programa de Prevención de la Violencia Escolar. Fundación Paz Ciudadana. Recuperado de http://www.pazciudadana.cl/docs/pub 20100218170450.pdf

Vargas, W. (2006). Atención Primaria de Salud en Acción: Su Contexto Histórico, Naturaleza y Organización en Costa Rica. San José, Costa Rica: EDNASSS-CCSS. Recuperado de http://www.binasss.sa.cr/libros/atencionprimaria.pdf

Yubero, S. (1996). El desafio de la educación social. La Mancha, España: Universidad de Castilla. 